\title{
Breathfinding: A Wireless Network that Monitors and Locates Breathing in a Home
}

\author{
Neal Patwari, Lara Brewer, Quinn Tate, Ossi Kaltiokallio, and Maurizio Bocca
}

\begin{abstract}
This paper explores using RSS measurements on the links between commercial wireless devices to locate where a breathing person is located and to estimate their breathing rate, in a home, while the person is sitting, lying down, standing, or sleeping. Prior RSS-based device-free localization methods required calibration measurements to be able to locate stationary people, or did not require calibration but only located people who moved. We collect RSS measurements multiple short (3-7 minute) tests and during a longer 66 minute test, and show the location of the breathing person can be estimated, to within about $2 \mathrm{~m}$ average error. We describe a detector that distinguishes between sample times during which a person is moving and sample times during which a person is breathing but otherwise motionless. This detector enables removal of motion interference, i.e., RSS changes due to movements other than a person's breathing, and more accurately estimate a person's breathing rate. Being able to locate and monitor a breathing person, without calibration, is important for applications in search and rescue, health care, and security.
\end{abstract}

\section{INTRODUCTION}

A significant body of research has shown that moving people can be located in a building using a static wireless network that measures links' RSS values [1], [2], [3], [4], $[5],[6],[7],[8],[9]$. This can be done through walls [6], [10], [11], [12], using a variety of statistics of the measured RSS. However, state-of-the-art RSS-based non-cooperative localization methods require either: 1) motion, that is, a person to be moving at least once during the course of the time in which measurements are collected [6], [13], [14], or 2) calibration, either measurements of the RSS from the period of time when the area was cleared of any people [3], [15], or measurements of the RSS while a person stands in each possible location [16], [8]. Even systems sophisticated enough to adaptively learn to distinguish the statistics of RSS during crossing vs. no crossing require some periods

Copyright (c) 2013 IEEE. Personal use of this material is permitted. However, permission to use this material for any other purposes must be obtained from the IEEE by sending a request to pubs-permissions@ieee.org. N. Patwari and M. Bocca are with the Department of Electrical and Computer Engineering, University of Utah, Salt Lake City, USA. N. Patwari is also affiliated with Xandem Technology. L. Brewer is with the Department of Anesthesiology, University of Utah Health Sciences Center, Salt Lake City, USA. Q. Tate is with the School of Medicine, University of Utah, Salt Lake City, USA. Ossi Kaltiokallio is with the Department of Automation and Systems Technology, Aalto University School of Electrical Engineering, Helsinki, Finland. This material is based upon work supported by the National Science Foundation under Grant Nos. \#0748206 and \#1035565. Contact email: npatwari@ece.utah.edu. of both to occur [17], [11]. Work on robotic RF sensor networks has demonstrated the ability to image concrete structures [18], however, work has not yet shown that a motionless person could be located without prior empty calibration.

This paper explores using standard wireless devices which measure only received signal strength (RSS) to monitor and localize the breathing of a person in a building, without any prior calibration. Radar devices can be used for this purpose [19], [20], but we believe that enabling breathing monitoring and localization using widely deployed commercial wireless devices may enable new low-cost systems, potentially making use of alreadydeployed wireless networking devices. Our work is the first, to our knowledge, to use RSS measurements to locate where breathing is occurring.

The core of the idea of RSS-based breathing localization is that a link's RSS measurements are sensitive to breathing when the breathing person is near the link line (the straight line between transmitter and receiver) and there is no other motion occurring nearby. From many links' RSS data, we can both estimate breathing rate and localize a breathing but otherwise motionless person.

We also address the challenge of keeping track of a person's breathing rate when the person occasionally moves. The same links that are sensitive enough to motion to change due to a person's inhalation and exhalation also experience motion interference, i.e., rapid change due to other movement, for example, if the person's limb moves. We show that using a change detector across all links, and negating the changes, allows the system to continue to estimate breathing rate in periods containing motion interference.

There are applications for RSS-based breathing monitoring and localization in health care, emergency response, and in context-aware computing. Existing breathing monitors used in health care typically require contact with the body. For example, a standard practice in hospitals is monitoring via a capnometer, which requires a patient to have a mask or nasal cannula attached to them [21]. Microwave Doppler radars are being commercialized for contact-free medical breathing monitoring [22], [23], [24]. Radar propagation losses are of order $d^{4}$ in free space [25], as opposed to $d^{2}$ for transmission [26], and thus radar devices either have limited range or high transmit power. Regarding the latter, UWB radar devices are severely limited in transmit power by regulation, and thus compliant devices have a low range. Although this paper does not 
provide human subject trials necessary for approval as a medical device, RSS-based monitoring could potentially be advantageous compared to radar-based monitoring.

One may be able to use a wireless network deployed in a bedroom as a baby breathing monitor, or as part of an in-home sleep apnea diagnostic system. We do not present algorithms to identify or distinguish the breathing rates of two people in the same room. However, breathing monitoring is most critical when the person is alone, and thus no other person is present to notice their apnea. Presumably a baby breathing monitor would be more important when no parent is in the same room as the infant, as studies indicate that SIDS rate is $50 \%$ lower when a parent sleeps in the same room as an infant [27].

We do not explore the localization of a breathing person trapped in a collapsed building, or lying unconscious due to smoke inhalation. However, RSS-based breathing localization could provide improved localization capabilities for emergency response applications because the system does not require either calibration or a person to move after the sensors are deployed.

In general, breathing rate provides a measure of a person's context, and context-aware computing systems could benefit. Perhaps "smart" homes could also be "empathetic" and respond appropriately when your at-rest breathing rate is abnormally high. A scary movie could adapt to the viewer's measured level of stress.

Our initial work was presented in [28], which introduced RSS-based breathing rate estimation and detecting the absence of breathing. The methods were experimentally verified on a subject in a hospital bed, using transceivers placed within centimeters of the subject's body. The report shows that links in a deep fade are the ones most likely to measure changes when the person breathes. Our breathing rate estimator is compared to the method of [28] in Section II-B.

This paper makes several contributions:

1) We show that a breathing but otherwise stationary person can be located in a building using RSS measurements on links in a wireless network, without any calibration or training. We provide a radio tomographic imaging method to show the breathing intensity in a deployment area, and locate the breathing person from the highest point in the image. We see average localization errors of about $2 \mathrm{~m}$. Our localization algorithm and experimental results are presented in Section IV-D.

2) Past work was capable of breathing rate estimation only when the person being monitored was completely stationary [28]. We introduce the use of "breakpoints" for mean removal, i.e., using change detection methods to determine when a person's movements alter link RSS values, setting these times as breakpoints, and subtracting from a link its average RSS during the period between two breakpoints. Using 30 seconds of data, our method's breathing rate estimates have average error of $1.0 \mathrm{bpm}$, compared to $1.7 \mathrm{bpm}$ for [28]. Our breathing rate estimation algorithm and experimental results are presented in Section II-B.

3) We use measurements on multiple frequency channels, and quantify the improvement possible compared to single-channel RSS measurements in Section IV-H. This paper is the first to demonstrate that breathing can be monitored using wireless networking devices meters, as opposed to centimeters, away from the person. Our testbed and experimental setups are described in Section III.

Finally, we conclude and discuss future research directions in Section V.

\section{Methods}

\section{A. Problem Formulation}

We assume there is a network of $S$ wireless devices which can operate on $C$ channels. Device $s$ is located at coordinate $z_{s}$. Link $l$ has transmitter (TX) $T_{l}$, receiver (RX) $R_{l}$, and channel $F_{l}$, i.e., each transmitter / receiver / channel combination is a different logical link. We denote the total number of measured logical links as $L$, that is, the total number of transmitter / receiver / channel combinations which the network measures. Full connectivity is not necessary for breathing monitoring.

We assume that each link's RSS is measured at regular intervals with period $T$ by sending and receiving a packet. We assume that there is both a maximum frequency $f_{\max }$ and a minimum frequency $f_{\min }$ at which breathing may occur. Note that newborns have the highest breathing rates, near 37 breaths per minute (bpm), or $0.62 \mathrm{~Hz}$, and adults at rest breathe near $10-14 \mathrm{bpm}$, or $0.23 \mathrm{~Hz}$ [29]. To sample at the Nyquist rate, sufficient for a newborn monitoring application, our sampling rate would need to be more than $1.24 \mathrm{~Hz}$. We denote the $n$th RSS on link $l$ as $r_{l}[n]$, in $\mathrm{dB}$ units. We further assume that the person is breathing at a constant rate. Future work could extend the presented algorithms for irregularly sampled data, and breathing which occurs irregularly, that is, with a period that changes over time. These assumptions limit the robustness of the method to random multiple access delays, and to erratic breathing patterns, respectively. We discuss the need for extensions of the proposed method in Section V.

The goals of the methods are: (a) for localization, to estimate the current coordinate of the breathing person; and (b) for rate estimation, to estimate $\hat{f}$, the rate at which the person repeats her inhalation and exhalation cycle.

\section{B. Breathing Rate Estimation}

1) Basic Method: In [28], an RSS-based breathing rate estimator is proposed which calculates the power spectral density (PSD) over each link using the most recent $N$ samples, sums the PSD over all links, and estimates the breathing rate as the frequency at the maximum of the sum PSD. Mathematically,

$$
\hat{f}=\underset{f_{\min } \leq f \leq f_{\max }}{\operatorname{argmax}} \sum_{l=1}^{L}\left|\sum_{n=i-N+1}^{i} y_{l}[n] e^{-j 2 \pi f T n}\right|^{2},
$$


where $i$ is the current time index, $j=\sqrt{-1}$, and $y_{l}[n]$ is the change in RSS compared to the mean, defined as

$$
y_{l}[n]=r_{l}[n]-\bar{r}_{l},
$$

where $\bar{r}_{l}$ is the average RSS on link $l$. We call $y_{l}[n]$ the $R S S$ signal because the mean RSS carries no information about breathing, and in fact, a strong DC component overwhelms any other frequency contained in the data. In [28], DC removal is performed using a 7-tap IIR filter. However, we find that setting $\bar{r}_{l}=\frac{1}{N} \sum_{n=i-N+1}^{i} r_{l}[n]$, i.e., the window average, has superior performance over a wide variety of experiments we have conducted. When comparing our method, to be fair to the spirit of the method in [28], we use this window average in (2).

We note that the function in (1) is a short-time Fourier transform when seen as a function of current sample index $i$. The window duration, $N$, must be chosen appropriately to the breathing pattern expected. If breathing may be erratic, i.e., changing period over short periods, then $N$ should be selected to be shorter, while if breathing is relatively constant, one may use a longer window $N$. In our experiments, we test using a 30 second window.

2) Breakpoint Method: One challenge faced by RSSbased breathing rate estimation is that the links which best measure the person's chest movement are also those that best measure other motion in the environment. This other motion, which we term "motion interference", can hide the breathing-induced changes. From our observation, motion interference and breathing cause notably different types of changes. While breathing causes slow, periodic RSS changes, motion interference typically causes sudden changes. For example, a person who rolls over in bed or moves a foot causes fast RSS changes during their movement. When the person stops moving, assuming they stop in a different position, a link's RSS typically settles to an average value different than before the movement. This is because the RSS is a very sensitive function of the position of objects in the environment of the link, which Woyach et. al. refer to as "spatial memory" [1].

If a large shift occurs during an $N$-sample time window, subtracting the $N$-sample average as in (2) will not suffice to remove the strong low-frequency component in the RSS signal induced by the shift. Depending on the magnitude of the shift, it may prevent the frequency-domain estimator in (1) from recovering the true breathing frequency. We note that the RSS signal with motion interference will be acceptable for localization, because a person can be located using existing DFL methods without calibration or training if they are in motion or at least occasionally in motion [6], [17], [11].

To make breathing rate estimation robust to motion interference, we propose to use change detection to identify each time index during which a sudden RSS change occurs. We call these detected time indices "breakpoints". Each breakpoint is a renewal point at which one window ends and the next window begins, and averages are calculated over each window. By doing so, we are able to perform more accurate mean removal, and thus make breathing rate estimation more robust to motion interference.

A variety of change-point detection methods, which have a long history of study in statistics and time-series analysis [30], may be useful for the task of identifying breakpoints. We require a change detector for a multivariate signal $\mathbf{r}=\left[r_{1}[n], \ldots, r_{L}[n]\right]^{T}$ for which we do not know a priori the distributions before and after the change. Note that the magnitude of the change is different on each link and unknown a priori, as shown in the example in Figure 3 in Section IV-A. Change detection methods which require known means or variances before or after the change point, such as multivariate versions of the cumulative sum (CUSUM) algorithm [31] or Bayesian change-point detectors [32], are not directly applicable to this problem. We believe a variety of change detection methods could be applied to identify breakpoints, and we test two in this paper.

First, we study using Welch's t-test for change detection. It is a generalized likelihood ratio test (GLRT) of the change in mean between two groups of normallydistributed data [33] when the two groups may also have different variances. In our case, one group of RSS signal samples is prior to the time-under-test (the sample index at which we want to detect a change in mean), and another same-duration group of data is after the time-under-test. Specifically, the group t-score is,

$$
\tau_{l}[n]=\frac{\grave{r}_{l}[n]-\grave{r}_{l}[n]}{\max \left\{\epsilon, \sqrt{\left(\grave{\sigma}_{l}^{2}[n]+\grave{\sigma}_{l}^{2}[n]\right) / Q}\right\}},
$$

for each link $l$ and time $n$, where $\grave{r}_{l}[n]$ and $\dot{r}_{l}[n]$ are the average of the $Q$ samples of $r_{l}[\mathrm{~m}]$ before and after time $n$, respectively, $\grave{\sigma}_{l}^{2}[n]$ and $\hat{\sigma}_{l}^{2}[n]$ are the sample variances of the $Q$ samples of $r_{l}[m]$ before and after time $n$, respectively, and $\epsilon>0$ is used to prevent division-by-zero. We use $\epsilon=0.5$ in our experiments, a choice examined in Section IV-B.

Note that we do not have evidence that the RSS data $r_{l}[m]$ are normally-distributed. In fact, quantization in the receiver and empirical measurements of temporal fading [34], [35] both provide evidence against Gaussianity.

The unknown distribution is motivation for a nonparametric detector. As a second method for change detection, we study using the Wilcoxon rank-sum test [36], perhaps "the most common non-parametric statistical test for change detection" [37]. This test can be more efficient than the t-test for data that is far from Gaussian [38]. The Wilcoxon rank-sum test sorts the $2 Q$ samples of $r_{l}[\mathrm{~m}]$ centered at time $n$ to compute the rank of each sample. Then, the ranks of the $Q$ samples before $n$ are summed, and the ranks of the $Q$ samples after $n$ are summed. We denote the maximum rank sum for link $l$ at time $n$ as $S_{l}[n]$, which is approximately Gaussian with mean $\mu_{S}=Q(2 Q+1) / 2$ and variance $\sigma_{S}^{2}=Q^{2}(2 Q+1) / 12[38]$. We compute a z-score $Z_{l}[n]=\left(S_{l}[n]-\mu_{S}\right) / \sigma_{S}$ which is thus a standard normal random variable. Intuitively, $Z_{l}[n]$ is high when the distributions of the samples before and 
after $n$ have significantly different means.

For either change detection method, we must combine the information across all links at time $n$ to make a single decision about whether there was a change in mean at that sample. We compute the root-mean-squared (RMS) average of $\tau_{l}[n]$ (for Welch's t-test) or of $Z_{l}[n]$ (for Wilcoxon's rank-sum test) over all links $l$,

$$
\begin{aligned}
& \tau_{R M S}[n]=\left(\frac{1}{L} \sum_{l=1}^{L} \tau_{l}^{2}[n]\right)^{\frac{1}{2}} . \\
& Z_{R M S}[n]=\left(\frac{1}{L} \sum_{l=1}^{L} Z_{l}^{2}[n]\right)^{\frac{1}{2}} .
\end{aligned}
$$

Time $n$ is a breakpoint index if $\tau_{R M S}[n] \geq \gamma_{t}$ (or $Z_{R M S}[n] \geq \gamma_{Z}$ ), where $\gamma_{t}$ or $\gamma_{Z}$ are predetermined thresholds. For any time window, we also consider the starting time index and ending time index as breakpoints. At the current time $i$, with a window length $N$, these indices are $i-N+1$ and $i$.

Finally, the breakpoint method we propose is to use the detected breakpoint indices to remove the mean from the raw RSS as follows:

$$
y_{l}[n]=r_{l}[n]-\tilde{r}_{l}[n],
$$

where $\tilde{r}_{l}[n]$ is the average of $r_{l}[m]$ for all $m$ such that $b_{p} \leq$ $m<b_{f}$, where $b_{p}$ is latest breakpoint before or at time index $n$, and $b_{f}$ is the earliest breakpoint after time $n$. Essentially, the mean model $\tilde{r}_{l}[n]$ is piecewise constant, with transition times at each breakpoint. After performing mean removal in (6), we use our new RSS signal $y_{l}[n]$ in (1) to perform breathing rate estimation.

\section{Breathing Localization}

In this section, we describe a method to compute a "map" of where the breathing, at the estimated rate, is occurring. From the map we can estimate the coordinate of the breathing person. In short, our method uses the breathing rate estimate $\hat{f}$ generated from either Section II-B1 or Section II-B2, and in particular, the amplitude of the signal component at $\hat{f}$ on each link, in order to estimate the person's location.

Variance-based radio tomographic imaging (VRTI) uses the variance of RSS on links in a wireless network to identify the location of a moving person in a building [6]. However, a breathing but stationary person does not show up in a VRTI image, as the image is more affected by noise than by the small changes caused by breathing. In this section, we adapt the approach from [6] for breathing mapping and localization, and we show using the experimental data that an approximate location of the breathing person can be determined.

We use the PSD at the estimated breathing rate for each link as an input to our method. For link $l$, at time $i$, the PSD of link $l$ at $\hat{f}$ is,

$$
v_{l}=\left|\sum_{n=i-N+1}^{i} y_{l}[n] e^{-j 2 \pi \hat{f} T n}\right|^{2} .
$$

The vector of all link values is $v=\left[v_{1}, \ldots, v_{L}\right]^{T}$. Note that $y_{l}[n]$ can be generated from either the basic or the breakpoint method.

We estimate an image of breathing amplitude vs. space, denoted vector $x$, where $x_{k}$ represents the quantity of breathing energy coming from pixel $k$. As in [6], we assume that $v$ is a linear combination of $x$ via a weighting matrix $W$ plus noise $\eta$,

$$
v=W x+\eta
$$

where $W$ is defined as having $(l, k)$ element given by,

$$
W_{l, k}=\left\{\begin{array}{ll}
\frac{1}{P_{l}}, & \frac{\left\|z_{T_{l}}-p_{k}\right\|+\left\|z_{R_{l}}-p_{k}\right\|}{\left\|z_{T_{l}}-z_{R_{l}}\right\|+\lambda_{e}} \leq 1 \\
0, & \text { o.w. }
\end{array},\right.
$$

where $z_{T_{l}}$ and $z_{R_{l}}$ are the coordinates of TX and RX for link $l$, respectively, $p_{k}$ is the coordinate of pixel $k, \lambda_{e}$ is the ellipse size parameter, and $P_{l}$ is the number of non-zero weights for link $l$. Essentially, $P_{l}$ normalizes the weight so that the total weight of each link is 1 . We note that as the path length $\left\|z_{T_{l}}-z_{R_{l}}\right\|$ increases, the ellipse becomes longer and thus increases in area, and thus the per-pixel weight $1 / P_{l}$ decreases.

Solving for $x$ in (8) given measurement $v$ is ill-posed. We assume the image vector has zero mean and covariance matrix $C_{x}=\sigma_{x}^{2} G$, where $G$ is a matrix with $(k, m)$ element $G_{k, m}=e^{-\left\|z_{k}-z_{m}\right\| / \delta}$, where $\sigma_{x}^{2}$ is the variance of any element of $x$, and $\delta$ is the correlation distance. This form of $C_{x}$ provides an exponential decay in correlation coefficient between pixels as a function of distance between the pixels. Such a spatial decay is an approximation of the covariance for a spatial random field whose value is the number of points of an underlying Poisson point process within a constant radius [39]. We then assume that the elements of the noise vector $\eta$ are independent and identically distributed, and thus the covariance matrix of $\eta$ is $\sigma_{n}^{2} I$, where $I$ is the identity matrix. We note that improved linear estimators can be developed when better noise models are available [40]. With these assumptions, an appropriate least squares estimator is,

$$
\begin{aligned}
\hat{x} & =\underset{x}{\operatorname{argmin}}\|W x-v\|_{\sigma_{n}^{2} I}^{2}+\|x\|_{C_{x}}^{2} \\
& =\underset{x}{\operatorname{argmin}} \frac{1}{\sigma_{n}^{2}}(v-W x)^{T}(v-W x)+x^{T} C_{x}^{-1} x .
\end{aligned}
$$

Taking the derivative of the argument of (10) and setting to zero to find $\hat{x}$,

$$
\hat{x}=\Pi v, \quad \text { where } \Pi=\left(W^{T} W+\frac{\sigma_{n}^{2}}{\sigma_{x}^{2}} G^{-1}\right)^{-1} W^{T} .
$$

Note $\Pi$ must be computed only once. The real-time computation of the image requires only one matrix multiply, of $\mathcal{O}(L P)$ multiplies and adds, where $P$ is the number of pixels. The ratio, $\sigma_{n}^{2} / \sigma_{x}^{2}$, essentially becomes a regularization parameter which can be tuned to emphasize either the prior on the image covariance or the measurement $v$.

From the image, the coordinate of the pixel with maximum value in $\hat{x}$ is used as the location estimate for the (single) breathing person. Future work must address localization in the multi-person case. 


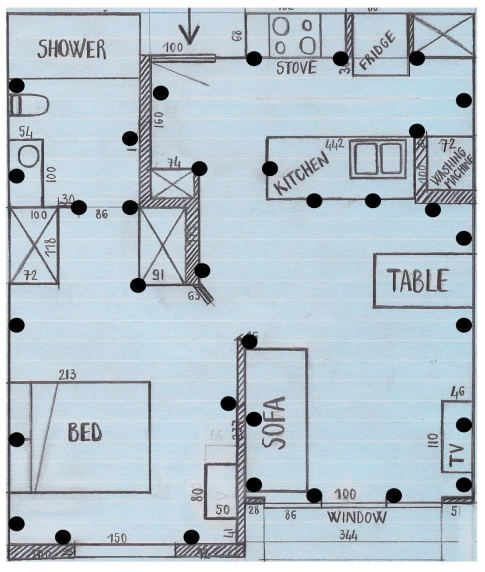

Fig. 1. Floor plan of $7 \times 8 \mathrm{~m}$ apartment, deployed nodes $(\bullet)$, and dimensions in $\mathrm{cm}$.

\section{EXPERIMENTS}

In this section, we describe the testbed network, the residential environment in which the tests are conducted, and the testing procedure.

\section{A. Network}

In our experiments, we use the TI CC2531 dongle node [41], an IEEE 802.15.4 compliant radio. The CC2531 has a transmit power of $4.5 \mathrm{dBm}$, and can select from one of 16 frequency channels in the $2.4 \mathrm{GHz}$ ISM band.

The network is a fully connected network, with each node able to transmit to and receive from every other node in the network. A multi-channel TDMA protocol is used so that node transmissions do not overlap. In this protocol, described in detail in [42], each node has a unique slot number, and transmits only during its slot. Nodes are in receive mode during all slots except for their own so that they hear the transmission from every other node. After a round of all nodes' slots are completed, nodes switch synchronously to the next frequency channel. A node's packet transmission includes its node ID and the RSS of the most recent packet received from each other node. A sink node that overhears all packets stores the data for post-processing.

\section{B. Apartment Experiment}

In our first experiment, the apartment experiment, we deploy $S=33$ nodes, each at a height of 0.9 meters. Our protocol uses $C=4$, specifically, 802.15.4 channel numbers $15,20,25$, and 26 , selected to overlap the least with WiFi. The center frequency of a 802.15.4 channel is given as $f_{c}=$ $2400+5(c-10) \mathrm{MHz}$, where $c$ is the channel number. Each link is measured each $T=428 \mathrm{~ms}$. This is a sampling rate of $2.3 \mathrm{~Hz}$.

The testbed network is deployed in the $7 \mathrm{~m}$ by $8 \mathrm{~m}$ apartment shown in Figure 1. Node locations are indicated with circles on the map. The apartment is a fully furnished residence on the third floor of a fully-occupied six story building in downtown Salt Lake City, Utah. Tests are

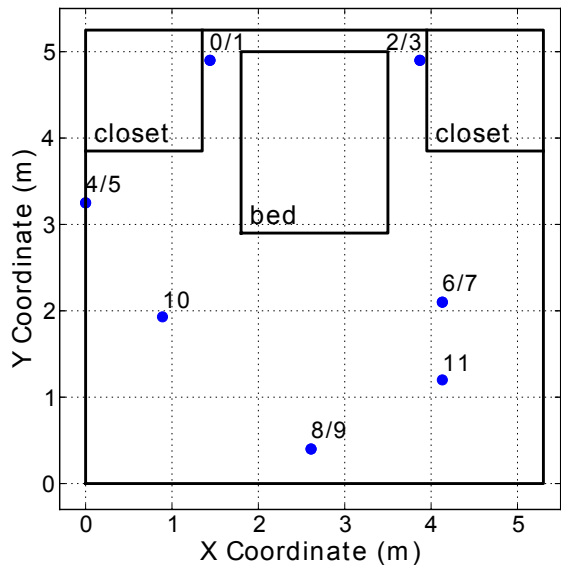

Fig. 2. Master bedroom floor plan, for "nap" experiment. Where two nodes are at the same $(x, y)$ coordinate on this $2 \mathrm{D}$ map, the higher node number is listed first.

conducted on a Saturday between noon and 1:00 pm. During the tests, other people are occasionally heard in the hallway outside of the apartment and in neighboring apartments.

As a large apartment building in a metropolitan area, there are many sources of $2.4 \mathrm{GHz}$ interference. The apartment has its own wireless router, and more than ten strong WiFi access points are observed, and although the channels are chosen to have the least overlap with WiFi, they are not perfectly orthogonal. Microwave ovens and cordless phones also exist in the nearby environment.

Tests: Five tests are conducted. In each, the experimenter stays in a single location for 3-7 minutes and maintains a constant breathing rate of $10 \mathrm{bpm}$ using a metronome. The experimenter is instructed to sit or stand at the location, but to move as required to be comfortable, i.e., move an arm or leg or change position. The experimenter made some movement, on average, twice per minute. In the tests, the experimenter is located: (1) standing in the kitchen; (2) sitting by the dining table; (3) sitting on the sofa; (4) lying in bed; and (5) sitting on the toilet.

\section{Nap Experiment}

Our second experiment, the nap experiment, is conducted in the master bedroom on the second floor of a house, as shown in Figure 2. $S=12$ nodes are deployed in a $5.3 \mathrm{~m}$ by $5.3 \mathrm{~m}$ area, at power outlet locations or in power strips in the room. We placed two nodes per power outlet, one on the floor and the other at a height of about 0.48 $1.65 \mathrm{~m}$. The height of the high nodes varied because they are placed on top of furniture (bedside table, dresser, table, etc.). In this experiment, $C=5$ channels are measured, specifically, IEEE 802.15.4 channels 11, 15, 18, 22 and 26. Here, the sampling period was $179.6 \mathrm{~ms}$, for a sampling rate of $5.56 \mathrm{~Hz}$.

There is also WiFi interference at this location, with one wireless router on the first floor below the bedroom. A laptop at location $(2 \mathrm{~m}, 0.5 \mathrm{~m})$ records data and sends it over WiFi to a server, ensuring WiFi interference to the breathing monitoring system. 


\begin{tabular}{|cc|}
\hline Parameter & Value \\
\hline Window Duration, $N$ & $70(30 \mathrm{sec})$ \\
Breathing Rate Min., $f_{\min }$ & $0.1 \mathrm{~Hz}(6 \mathrm{bpm})$ \\
Breathing Rate Max., $f_{\max }$ & $0.4 \mathrm{~Hz}(24 \mathrm{bpm})$ \\
Nodes, $S$ & 33 \\
Channels, $C$ & 4 \\
Links, $L=C S(S-1)$ & 4224 \\
Sampling Period, $T$ & $0.428 \mathrm{sec}$ \\
Change Det. Window, $Q$ & $14(6 \mathrm{sec})$ \\
RMS t-test Threshold, $\gamma_{t}$ & 0.8 \\
RMS Wilcoxon Threshold, $\gamma_{Z}$ & 1.15 \\
\hline
\end{tabular}

TABLE I

Breathing Estimator and Apt. Experiment Parameters

A 66-minute long "person sleeping" test is conducted. The experimenter starts collecting data on the laptop, walks to and climbs into the bed, and takes a nap. In contrast to the previous experiments, the experimenter's breathing rate is not known, as no separate breathing monitoring sensor is attached to the person, and the person cannot deliberately maintain a constant breathing rate while sleeping. After time 65 minutes, the person wakes up, gets out of bed and stops the data recording. The person moved at least once during sleep, since it was noted that he did not wake up in the same position in which he went to sleep.

A second "no person" test is conducted with the experimenter out of the room (about $10 \mathrm{~m}$ away) for a duration of three minutes. This second test is performed to be able to see how the RSS data, when no breathing person is present, compares to the case when the person is present and breathing in the room.

\section{Results}

\section{A. Breathing Rate: Apartment Experiment}
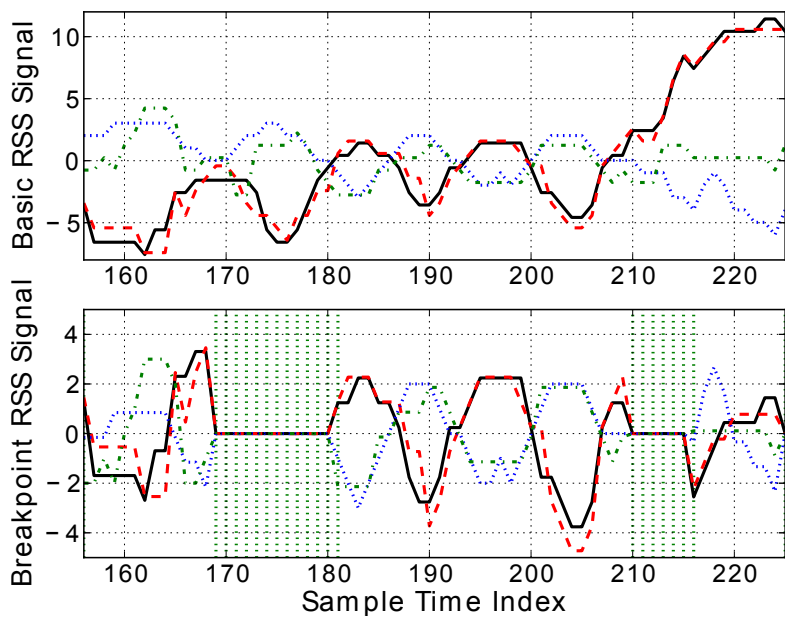

Fig. 3. The mean-removed RSS signal $y_{l}[n]$ for four links that best show breathing during 'sofa' experiment, using (top) basic and (bottom) breakpoint methods, with estimated breakpoints (:).

To compare the basic and breakpoint methods, we describe an example taken from a 30 -second window during the 'sofa' experiment. Methods are tested using parameters given in Table I. Figure 3 shows the mean-removed

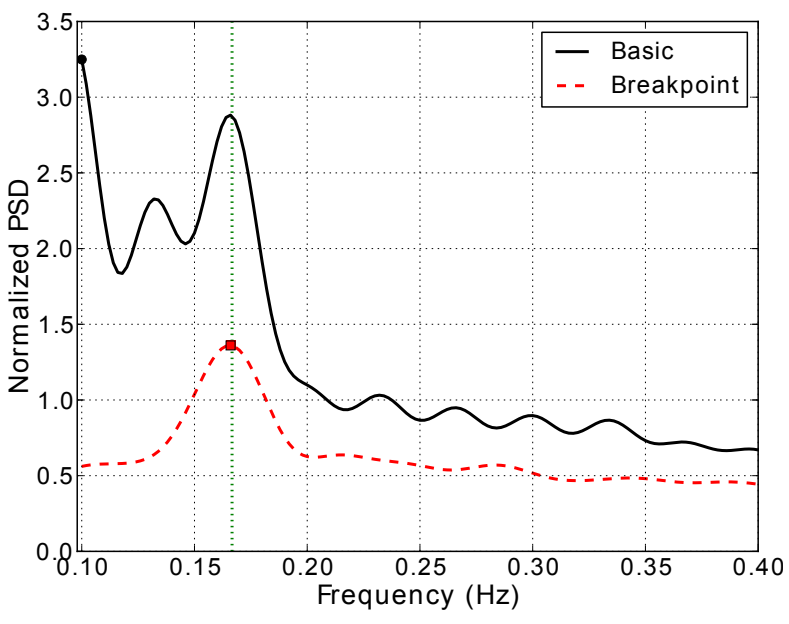

Fig. 4. Normalized average PSD from (1) using basic and breakpoint methods and data from Figure 3, estimated breathing rate $(\bullet$ and $\square)$, and true breathing rate $(:)$.

RSS signal $y_{l}[n]$ for the basic and t-test breakpoint methods, for four links $l$ that are the four best in terms of signal amplitude at the true breathing frequency, $v_{l}$. Note that the methods use $L=C S(S-1)=4224$ in this case, but we that we are plotting only four links for clarity. In the basic method, $y_{l}[n]$ drifts upward for two of the links, particularly around $n=213$. Many links (including those not shown) exhibit a shift in mean at this time index, as well as near $n=175$. The t-test breakpoint method, using a threshold $\gamma_{t}=0.8$, calculates a high RMS t-score near these two times, and detects several time indices near 213 and 175 as breakpoints. By removing the mean in between each pair of breakpoints, the signal $y_{l}[n]$ no longer has a drifting mean in the t-test breakpoint method. Note that some ranges are reduced to zero because breakpoints are dense, and removing the average from a single sample results in a zero value.

We note that in Figure 3, some links increase in RSS while some decrease in RSS. An explanation given for this in [28] is that the breathing-induced signal is the result of a phasor sum of paths, and when one phasor's phase changes as a result of breathing, it may increase, decrease, or leave unchanged the magnitude of the phasor sum. Thus when a person inhales, they may increase the RSS on some links, while simultaneously decreasing the RSS on other links.

Figure 4 shows the t-test breakpoint method enables correct breathing rate estimation when the basic method is unable. The basic method's PSD is shown to be highest at the lowest breathing rate, $0.10 \mathrm{~Hz}$, and thus $\hat{f}=0.10$ $\mathrm{Hz}$. The PSD for the breakpoint method is reduced at all frequencies, but has a clear maximum at $0.166 \mathrm{~Hz}$, very close to the true $0.167 \mathrm{~Hz}$ breathing rate.

Next we test all 30 second windows for all test locations, moving the window 5 seconds each time. We first report the fraction of estimates $\hat{f}$ which are acceptable, which we arbitrarily define as being within $3.0 \mathrm{bpm}$ of correct. Second, we report the average frequency error over all windows. Results are given in Table II for the basic and 


\begin{tabular}{|c|c|c|c|c|c|c|}
\hline \multirow[b]{3}{*}{ Loc. } & \multicolumn{3}{|c|}{ \% Acceptable } & \multicolumn{3}{|c|}{ Avg. Err. (bpm) } \\
\hline & Basic & Brea & point & Basic & Bre & point \\
\hline & & Wilc. & t-test & & Wilc. & t-test \\
\hline Sofa & $71 \%$ & $94 \%$ & $94 \%$ & 1.23 & 0.345 & 0.348 \\
\hline Table & $81 \%$ & $93 \%$ & $89 \%$ & 1.14 & 0.712 & 0.831 \\
\hline Kitchen & $58 \%$ & $77 \%$ & $91 \%$ & 1.81 & 2.11 & 0.859 \\
\hline Bath & $71 \%$ & $97 \%$ & $97 \%$ & 1.29 & 0.328 & 0.339 \\
\hline Bed & $25 \%$ & $35 \%$ & $33 \%$ & 2.95 & 2.64 & 2.63 \\
\hline Avg. & $61 \%$ & $79 \%$ & $81 \%$ & 1.69 & 1.23 & 1.00 \\
\hline
\end{tabular}

TABLE II

BASIC VS. BREAKPOINT BREATHING RATE ESTIMATION: PERCENT OF ESTIMATES "ACCEPTABLE", AND AVERAGE ERROR OF ACCEPTABLE ESTIMATES. BREAKPOINTS ARE DETERMINED EITHER BY THE WELCH'S T-TEST OR WILCOXON RANK-SUM CHANGE DETECTION METHODS.

breakpoint method, where the breakpoints are found using either the t-test or the Wilcoxon rank-sum test. Note that the threshold for the Wilcoxon test, $\gamma_{Z}=1.15$, is chosen so that the Wilcoxon change method detects approximately the same number of breakpoints as the t-test.

Results are shown in Table II. Over all tests, the basic method obtains an acceptable rate estimate $61 \%$ of the time. The breakpoint method achieves $79 \%$ acceptable and $81 \%$ acceptable estimates with the Wilcoxon and t-test change detection methods, respectively, approximately cutting in half the number of 'unacceptable' rate estimates. With the t-test breakpoint method, four of five tests have acceptable rates above $89 \%$, whereas the basic method had none. The t-test breakpoint method reduces the average breathing rate error to $1.0 \mathrm{bpm}$, a $41 \%$ reduction from the basic method. Primarily, the reduction in average error comes from eliminating large errors both methods typically have less than $0.3 \mathrm{bpm}$ error when the rate estimate is "acceptable". For reference, an endtidal $\mathrm{CO}_{2}$ meter, the gold standard breathing rate monitor in hospitals [21], is accurate to $\pm 1 \mathrm{bpm}$.

We note that the performance of the t-test and Wilcoxon rank-sum test are very similar. Both are significantly better than the basic method. The best average performance across these five tests is using the t-test. As a result, in the rest of this section, we report results when using the t-test in the breakpoint method.

The bed test has particularly poor performance. If we had used the t-test breakpoint method with $\gamma_{t}=0.5$ (instead of 0.8), the bed test would have had $73 \%$ acceptable estimates (vs. 33\%), however, the kitchen test would perform worse with the lower threshold. Future work should address adaptive methods to set the threshold.

\section{B. Parameter Sensitivity}

In this section, we study the performance of breathing rate estimation as a function of two of the Welch's ttest parameters, $\epsilon$ and $\gamma_{t}$, which we show have similar characteristics.

The use of $\epsilon$ is motivated because some links, particularly in an area with little motion, measure the same RSS value at almost every time index. Note that the measured RSS value is quantized, in our transceivers, to the nearest 1
$\mathrm{dBm}$, so the probability that $r_{l}[n]=r_{l}[n+1]$ has a positive probability. On these relatively stable links, a set of $Q$ samples sometimes has a variance of zero, or very close to zero, which result in infinite or very high t-scores in (3). The problem is that, because of the quantization of RSS, these links would estimate a standard deviation of zero, even though the standard deviation of the unquantized RSS would not have been zero, if it could have been calculated.

To test the sensitivity of performance to $\epsilon$, We test, for each apartment experiment, the percentage of estimates acceptable, as a function of $0<\epsilon<1$, and plot the results in Figure 5(a). We see that below a minimum $\epsilon$, approximately 0.25 in Figure 5(a), performance is very poor. This is very close to the minimum positive group standard deviation that is possible to be measured in our case - if all of the RSS values are identical in the two $Q$-sized samples except for one RSS value that is different by one, the calculated group standard deviation $\sqrt{\left(\grave{\sigma}_{l}^{2}[n]+\hat{\sigma}_{l}^{2}[n]\right) / Q}$ is 0.26 for $Q=14$. We also see that as $\epsilon$ is made too high, beyond 0.65 in the average plot in Figure 5(a), the performance of the estimator degrades, as fewer breakpoints are detected. As $\epsilon \rightarrow \infty$, no breakpoints will be detected, and the method reverts to the basic method.

(a)
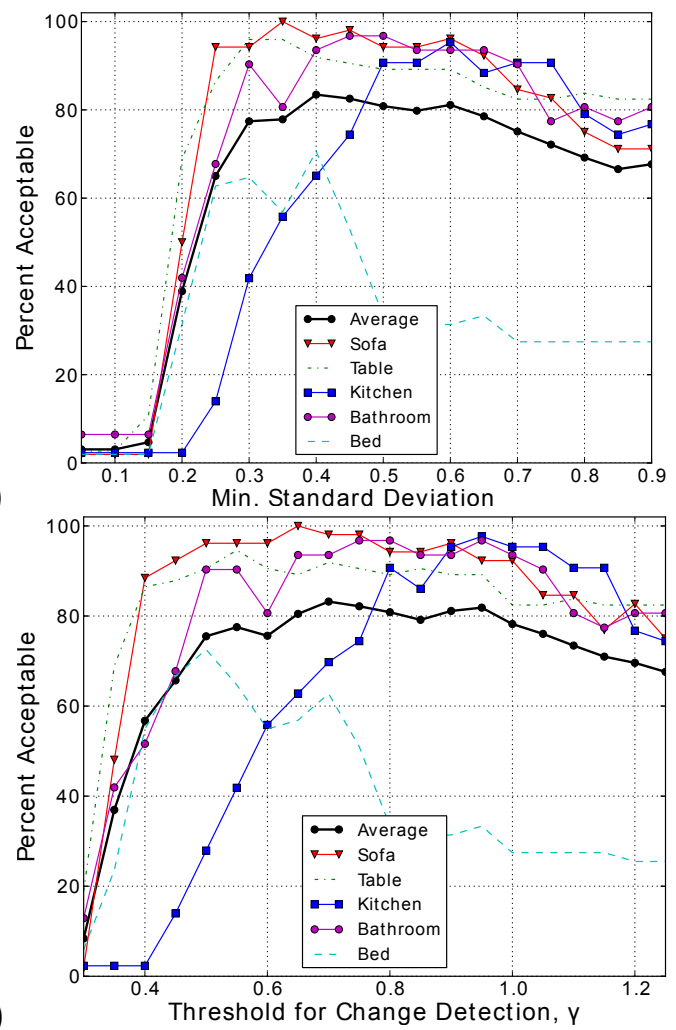

Fig. 5. Breakpoint method performance (percent rate estimates "acceptable") vs. parameters (a) $\epsilon$, and (b) $\gamma_{t}$; for each apartment data set, and averaged over the five data sets. In (a), $\gamma_{t}=0.8$, and in (b), $\epsilon=0.5$.

Next, we study the sensitivity of breathing rate estimation to the threshold $\gamma_{t}$. Recall that if $\tau_{R M S}[n]>\gamma_{t}$ then time index $n$ is included as a breakpoint. Using 


\begin{tabular}{|cc|}
\hline Parameter & Value \\
\hline Window Duration, $N$ & $167(30 \mathrm{sec})$ \\
Breathing Rate Min., $f_{\min }$ & $0.1 \mathrm{~Hz}(6 \mathrm{bpm})$ \\
Breathing Rate Max., $f_{\max }$ & $0.4 \mathrm{~Hz}(24 \mathrm{bpm})$ \\
Nodes, $S$ & 12 \\
Channels, $C$ & 5 \\
Links, $L=C S(S-1)$ & 660 \\
Sampling Period, $T$ & $0.1796 \mathrm{sec}$ \\
t-test Window, $Q$ & $14(2.5 \mathrm{sec})$ \\
RMS t-score Threshold, $\gamma_{t}$ & 0.8 \\
\hline
\end{tabular}

TABLE III

Breathing Rate Estimator and Nap Experiment Parameters.

each location from the apartment experiment, we test a range of $0.3 \leq \gamma_{t} \leq 1.25$ in 0.05 increments, and plot the results in Figure 5(b). The average performance, in terms of percent of estimates acceptable, is reasonably constant for $\gamma_{t}$ between 0.6 and 1.0. As in the study of $\epsilon$, as $\gamma_{t} \rightarrow 0$, all indices are detected as breakpoints, and the performance degrades. As $\gamma_{t} \rightarrow \infty$, no points are detected as breakpoints, and the performance reverts to the basic method.

For both $\epsilon$ and $\gamma_{t}$, most data sets have very similar performance, but the kitchen and the bed tests have significantly different optima. Future work should address adaptively setting these parameters based on the situation.

\section{Breathing Rate: Nap Experiment}

The data from the nap experiment is processed with the t-test breakpoint method, using the parameters given in Table III. Note these are identical to the apartment experiment except that $S=12$ nodes and $C=5$ channels are used, and because of that, the number of links is smaller, and sampling period is lower.

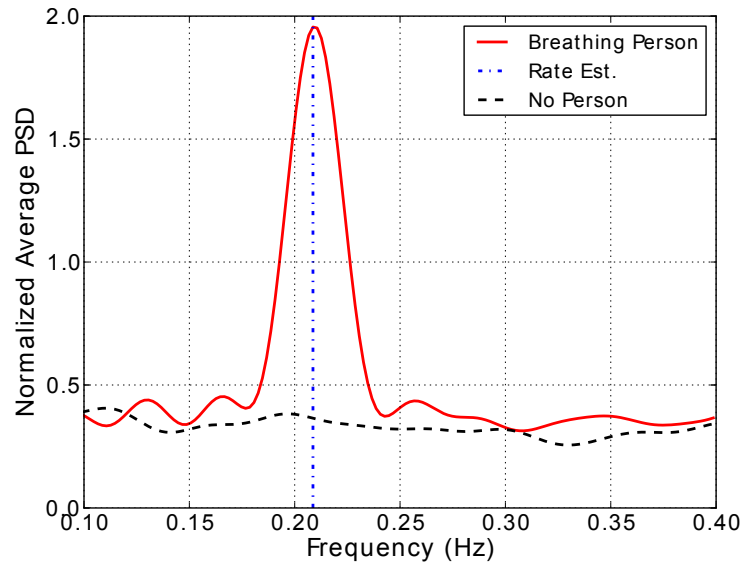

Fig. 6. Average normalized PSD of (1) when a breathing person is present $(-)$ with rate estimate $(-\cdot-\cdot-)$ compared with when no person is present (- - - -).

First, for the t-test breakpoint method, we compare the "no person" condition with the "person sleeping" condition. Figure 6 shows the average normalized PSD of (1) for a typical 30-second period of the "no person" test along with that of a typical 30-second period from the "person sleeping" test. The PSD is significantly lower when no person is

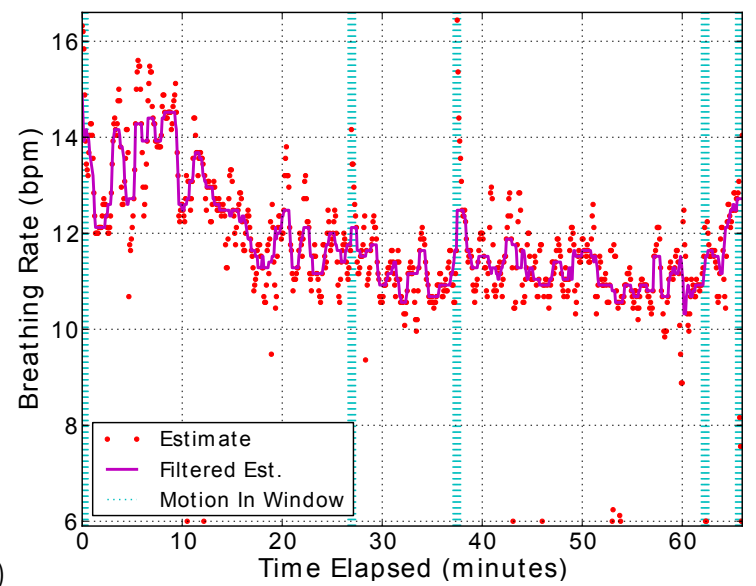

(a)

(b)

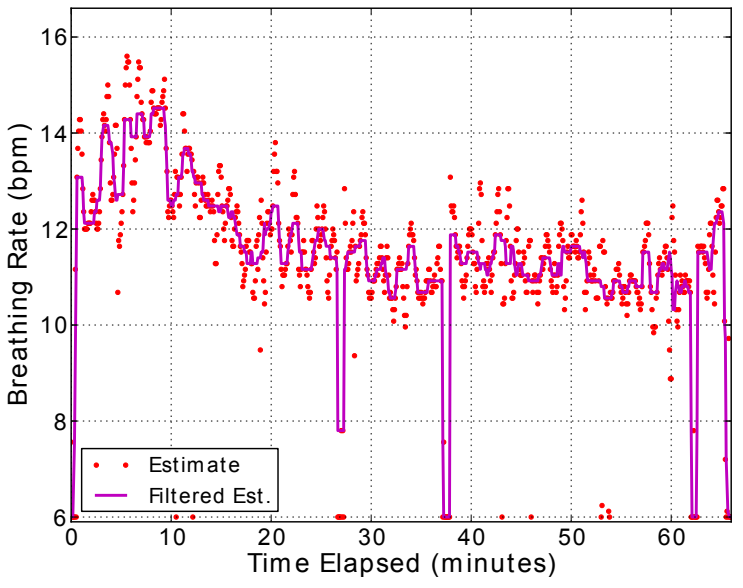

Fig. 7. Nap experiment breathing rate estimates based on $30 \mathrm{~s}$ data window $(\cdot)$, and $90 \mathrm{~s}$ median (-), for: (a) breakpoint with periods

of identified motion (:); and (b) basic method.

present in the room. We note that other 30 second periods in each condition also support the observation that the PSD has a peak with a significantly higher value when a breathing person is present, compared to when no person is present. A similar observation is made in [28] using significantly more "no person" tests, and the maximum amplitude of the PSD in (1) is used to reliably detect whether or not a breathing person is present or not.

As discussed in Section III-C, the true breathing rate is not known for the nap experiment, however, there are features of the breathing rate estimates from the breakpoint method, shown in Figure 7(a), which match our understanding of the human body during sleep. It has been shown that breath rate decreases during the transition from the awake state to stage two of sleep [43]. This is primarily because there is a downward trend in energy expenditure during the onset of sleep [43], [44]. The breath rate in the nap experiment decreases approximately $20 \%$ in the first 20 minutes, which correlates with published data showing a measured metabolic rate decrease of approximately $20 \%$ during the first 20 minutes of the onset of sleep [44]. During sleep stages 2, 3/4 and REM (rapid eye movement), the ventilation and metabolic rate are known to become relatively stable [43], [45], which is 
consistent with what we observed in our nap experiment estimates. However, moving during sleep increases energy expenditure and thus could temporarily increase breathing rate [43], as we see clearly at time 38 minutes, just after a period in which motion is detected Welch's t-test. At the end of the nap experiment, the breath rate increased as the person awoke without an alarm. This is consistent with published data showing increased movement and metabolic rate during the transition to wakefulness [44].

In a period containing motion that is not detected and removed by the breathing rate estimation algorithm, the rate estimate tends to rail to $f_{\min }$. This is because a single step function, when the step is large compared to other signals, would be estimated to be evidence of one cycle in the measurement period. For a 30 second window, this would be a rate of $2 \mathrm{bpm}$. Since $f_{\min }=6 \mathrm{bpm}$ in these experiments, the estimator will return a value of 6 $\mathrm{bpm}$ in this case. Step functions are not removed when using the basic algorithm, and in some instances, the t-test breakpoint method may not detect a step function if the amplitude of the change is small compared to the variances before and after the jump. Although we are not certain of the sleeping person's true breathing rate, we quantify the number of rate estimates at or very near $6 \mathrm{bpm}$ as a measure of the ability of the method to remove normal (non-breathing) motion from the breathing-induced signal. In this experiment, using the t-test breakpoint method, there are fourteen breathing rate estimates below $9 \mathrm{bpm}$, five of which are during identified periods of motion. Thus out of 793 window $\hat{f}$ estimates, only nine $(1.1 \%)$ are very low and are during periods when no motion is detected.

In comparison, we show results for the basic meanremoval method in Figure 7(b). When using the basic method, two metrics are worse. First, there are 48 estimates with rate below $9 \mathrm{bpm}$. Since there are several of these low estimates in a row, the median filter also falls below $9 \mathrm{bpm}$ during periods that we believe (from the t-test breakpoint method results) contain movement. In contrast, the median filter in the breakpoint method never falls below $9 \mathrm{bpm}$. Not only are the basic method rate estimates presumably incorrect, in addition, there is no "motion" label provided to indicate to the application that there was likely motion in that period. As a result, there is no way to automatically discard the poor rate estimates returned by the basic method.

A monitor that estimates very low or very high breathing rates, particularly if repeated, would raise an alarm in many applications. The reason for breathing monitoring is often to alert a professional when emergency medical attention is needed. The system using the basic meanremoval method would, in this experiment, raise several false alarms during the hour-long time period, and waste time and resources by bringing medical attention to a patient when she was breathing normally.

\section{Breathing Localization: Apartment Experiment}

In this section, we implement the breathing mapping and localization of (11) using the parameters given in Ta-

\begin{tabular}{|lc|}
\hline Parameter & Value \\
\hline Pixel Width, $\delta_{p}$ & $0.2 \mathrm{~m}$ \\
Variance Ratio, $\frac{\sigma_{n}^{2}}{\sigma_{x}^{2}}$ & 0.5 \\
Correlation Distance, $\delta$ & $2 \mathrm{~m}$ \\
Ellipse Size Parameter, $\lambda_{e}$ & $1 \mathrm{~m}$ \\
\hline
\end{tabular}

TABLE IV

BREATHING MAPPING AND LOCALIZATION PARAMETERS

\begin{tabular}{|l|cc|}
\hline & \multicolumn{2}{|c|}{ Avg. Loc. Error (m) } \\
Test Loc & Basic & Breakpoint (t-test) \\
\hline Sofa & 1.5 & 1.7 \\
Table & 2.1 & 2.1 \\
Kitchen & 2.9 & 3.6 \\
Bathroom & 1.5 & 1.9 \\
Bed & 2.6 & 2.7 \\
\hline Average & 2.1 & 2.4 \\
\hline
\end{tabular}

TABLE V

Average LOCATION ERRORS FOR BASIC AND BREAKPOINT METHODS

ble IV, and evaluate its performance using the apartment experiment.

We note that parameters in Table IV are not necessarily optimal. The pixel width $\delta_{p}$ will determines $P$, the number of pixels in the image. Image estimation has computational complexity $\mathcal{O}(P)$, it is desirable to set $\delta_{p}$ high, but quantization errors increase with increasing $\delta_{p}$. The value of $\delta_{p}=0.2 \mathrm{~m}$ was chosen to be smaller than the best case average error we might possibly expect. We used a larger ellipse size $\lambda_{e}=2 \mathrm{~m}$ than used in either [42] or [6]. This is because we know that links that detect breathing tend to be those with average or lower-than average RSS for their path length [28], and that such links are affected by motion in a wide area around the link line [9]. Finally the two regularization parameters, $\delta$ and $\frac{\sigma_{n}^{2}}{\sigma_{x}^{2}}$, were set by trial-and-error, we simply ran several settings and checked performance on the apartment data sets. Ideally, parameters should be set based on those used in prior tests in similar environments, and would not need to be tuned for a particular deployment. For example, localization for the nap experiment was performed using the same parameters as determined for the apartment experiment, even though it had a different number of sensors and was conducted in a different home.

Using the same example window as that shown in Figure 3 , we first draw link lines corresponding to the few links $l$ with highest $v_{l}$ in Figure 8(a), where $v_{l}$ is the PSD of link $l$ at the breathing rate estimate, as defined in (7). These include the four plotted in Figure 3. The image $\hat{x}$ for the same data is shown in Figure 8(b). For this example, the location estimate has $0.82 \mathrm{~m}$ error.

We summarize location estimation performance in the apartment experiment in Table V. Using the basic method for mean removal, the average location error over all tests is $2.1 \mathrm{~m}$. In a 7 by 8 meter area, this is a coarse estimate relative to performance reported for radio tomographic imaging [6], [42], however, it is typically sufficient to tell which room the person is in. For the t-test breakpoint method, the localization estimates degrade $14 \%$ to $2.4 \mathrm{~m}$. 
(a)

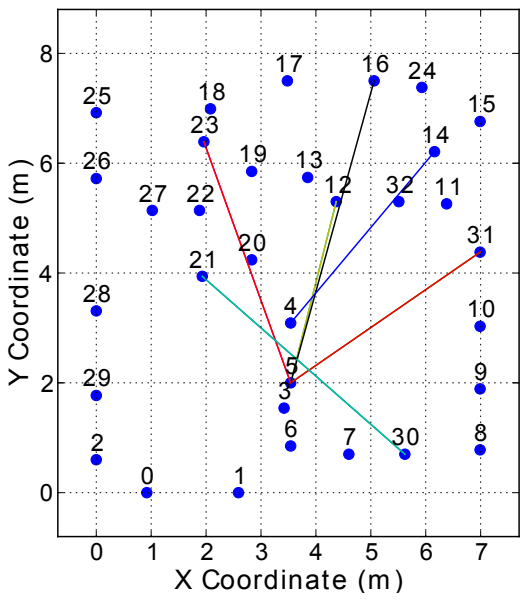

(b)

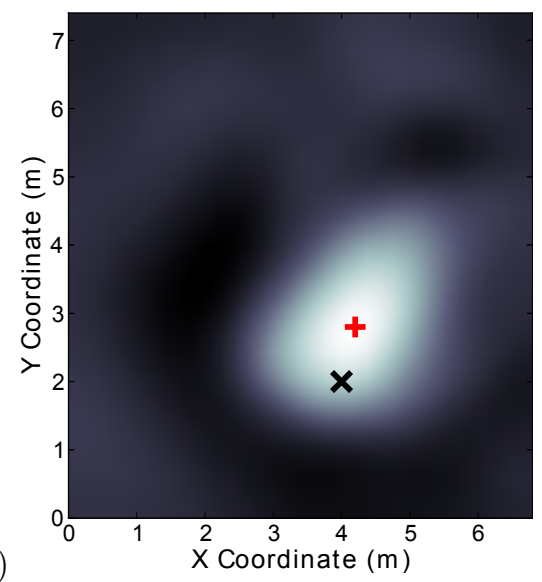

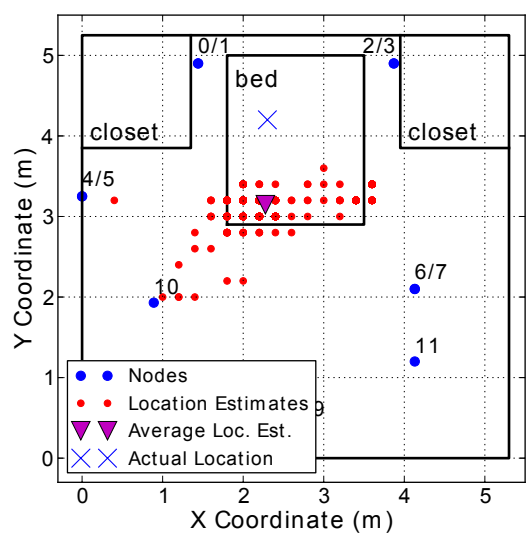

Fig. 8. Apt. "couch" exp.: (a) lines for the top ten links by $v_{l}$, and (b) breathing image $\hat{x}$ (white=high), with true $(\times)$ and estimated $(+)$ locations. In (c), location ests. for each 30 second window (of which there are 792) during the nap experiment.

From examination of the RSS data and images produced using the basic method for mean removal, we see that when windows include motion interference caused by the experimenter, the breathing map $\hat{x}$ is as accurate or more accurate than without the motion interference. This is because the experimenter is causing the motion, which then leaks into the RSS signal on links near the experimenter, and helps to increase the image value near the experimenter's location. The breakpoint method removes most motion interference, which then decreases the image value near the correct location.

We notice some times when the image suddenly shifts and shows a bright spot at the top of the apartment, where there is a hallway outside of the apartment wall. The experimenter had heard people walking through the hallway during some tests. However, we did not record the actual times of these events and thus future controlled experiments are suggested.

\section{E. Breathing Localization: Nap Experiment}

Finally, we test the nap experiment data for localization performance. We use the same algorithm (using the ttest breakpoint method) and same parameters (shown in Table IV). There is only one actual coordinate, which we measure to be the position of the chest when the person started napping. There are 792 estimates, which are shown in Figure IV-E. Since we use the center of the maximum pixel of $\hat{x}$ as the location estimate, many of the location estimates exactly overlap. We also show the average of all location estimates in Figure IV-E. This average is about $1.0 \mathrm{~m}$ away from the true chest position. Considering the estimates individually, the RMS localization error is 1.2 $\mathrm{m}$. In general, performance is better than the average of the apartment experiment, but the size of the area is also smaller as well, about half of the area of the apartment.

\section{F. Discussion}

It is interesting to note that both experiments have a "lying in bed" condition. In the apartment experiment, the person is lying in bed, awake, breathing at a constant rate, while in the nap experiment, the person is sleeping in the bed without a controlled rate. The localization performance is significantly better in the nap vs. the apartment experiment (1.0 m vs. $2.7 \mathrm{~m}$ average error), even though the node density $\left(0.42\right.$ nodes $/ \mathrm{m}^{2}$ in the nap experiment, 0.59 nodes $/ \mathrm{m}^{2}$ in the apartment experiment) is similar. Although the true breathing rate is not known for the nap experiment, we believe that the breathing rate estimates are "acceptable" significantly more often than $33 \%$, as was the case in the "bed" location in the apartment experiment. Among differences between the two experiments, one significant one is the use of sensors at different heights in the nap experiment, whereas the apartment experiment nodes were all uniformly placed at a height of $0.9 \mathrm{~m}$. Due to the $0.9 \mathrm{~m}$ node height in the apartment experiment, the link lines passed purely above the person in the bed. In contrast, some of the link lines between nodes at different heights in the nap experiment crossed through the sleeping person, and presumably diffracted around the person's body. Some of the links with highest $v_{l}$, as defined in (7) are those links which cross through the sleeping person.

Finally, computation time is about $0.2 \mathrm{~s}$ per $30 \mathrm{~s}$ window, for both rate estimation and localization, in Python on a two-core $2.0 \mathrm{GHz}$ processor - thus real time monitoring is very possible. There is some non-causality in the monitoring method: 1 ) we estimate the breathing rate after the window of data ( $N$ samples) is completely collected; 2 ) The t-test requires $Q$ samples, as described after (3), requiring additional delay. In our tests, $Q=14$ samples (6 seconds in the apartment experiment and 2.5 seconds in the nap experiment), thus the breakpoint method adds a few seconds of latency, depending on the sample rate. In contrast, the basic method adds no latency beyond the data window $N$. 


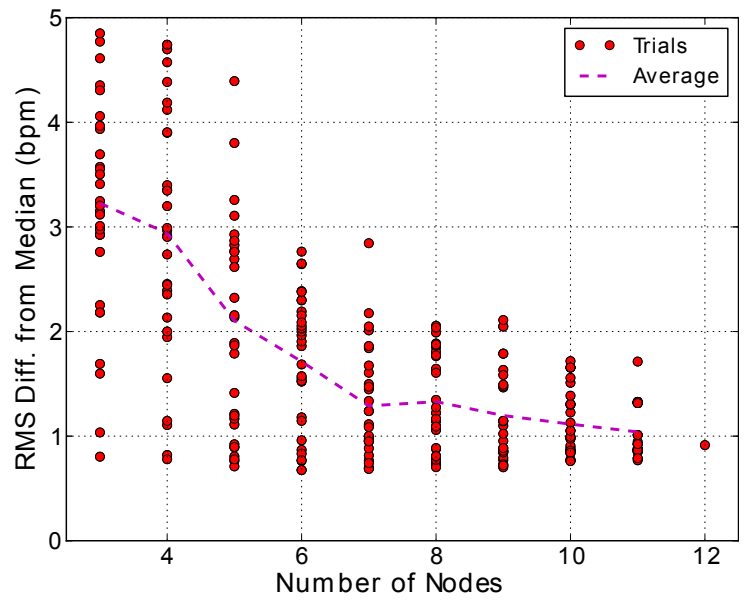

Fig. 9. RMS of the difference between the estimate and the shortterm median, as a function of $S$, the number of nodes, for nap experiment.

\section{G. Number of Nodes}

In this section, we show the performance of breathing monitoring when we only use a subset of nodes, to test using experimental data how the performance changes as a function of $S$. We would like to know, if the person's breathing is only to be monitored in one area (and not an entire home), how many nodes are required for acceptable performance? The nap experiment has all nodes in the bedroom, the same room as the experimenter was located. In this section, we both randomly and deterministically chose subsets of the twelve nodes used in the experiment and test performance only using that subset of nodes.

Since the true breathing rate is not known during the nap experiment, we evaluate the performance of breathing rate estimation as follows. We assume that short-term variation in breathing rate estimates are characteristic of a less-accurate estimator. As such, we report the RMS difference between a current breathing rate and the shortterm median, where the median is taken from all breathing rates estimated in a 90 -second window centered at the current time, which we refer to as the "RMS-median".

First, we test using random subsets of the twelve nodes. For each number of included nodes $S<12$, we run 30 random trials. We show the RMS-median value for each trial, as well as the average vs. $S$, in Figure 9 . The RMSmedian value, on average, decreases with $S$; however, the lowest possible RMS-median value seems to have a shallow minimum at a value of $S=6$, and every tested subset size had some trial with RMS-median value below 1.0. It would seem that low number of nodes is fine if you select the right nodes.

To explore this further, we test specific (non-random) subsets of nodes and compare the results in Table VI. First, we have eight nodes near the four corners of the bed, with nodes $0,2,4$, and 6 at a height of about 0.67 $\mathrm{m}$, and nodes $1,3,5,7$ on the floor. We first test the four nodes on the floor, $\{1,3,5,7\}$, and see an RMS-median of $1.96 \mathrm{bpm}$. In contrast, the four nodes at $0.67 \mathrm{~m},\{0,2,4,6\}$

\begin{tabular}{|cc|}
\hline Subset of Nodes & RMS-median (bpm) \\
\hline$\{0,2,4,6\}$ & 0.72 \\
$\{1,3,5,7\}$ & 1.96 \\
$\{0,2,4,6,8\}$ & 0.84 \\
$\{0,1,2,3\}$ & 3.57 \\
$\{0,1, \ldots, 5\}$ & 1.31 \\
$\{0,1, \ldots, 7\}$ & 0.68 \\
$\{0,1, \ldots, 9\}$ & 0.73 \\
$\{0,1, \ldots, 11\}$ & 0.91 \\
\hline
\end{tabular}

TABLE VI

RMS-MEDIAN USING NODE SUBSETS

have RMS-median of $0.72 \mathrm{bpm}$. We suspect that the links between nodes on the floor have signals which propagate largely along the floor, and as such do not interact strongly with the person in the bed. We also test using nodes $\{0,1,2,3\}$, the nodes closest to the person. Using this set of nodes results in a very high RMS-median of $3.57 \mathrm{bpm}$. Although they are close to the person, they are close to the person's head, and no link line crosses the person's chest.

\section{H. Number of Channels}

In prior work [28], nodes used only one channel. We are interested in whether our performance has improved due to the measurement of multiple channels. Here, we test the performance during the nap experiment when only links from a subset of the channels are used. We test each possible (non-empty) subset of the five measured channels, and plot the RMS-median performance in Figure 10. First, using all $S=12$ nodes, we see average performance improves as the number of utilized channels increases, although slowly. At $C=1$, the average RMS-median is $1.56 \mathrm{bpm}$, while when using all $C=5$ channels, the value is 0.91 , a $41 \%$ reduction. The result is much more pronounced when re-running the test using only the nodes $\{0,2,4,6\}$. These were a set of four sensors at height 67 $\mathrm{cm}$ closest to the bed, which achieve a RMS-median of 0.83 when using all $C=5$ channels. The improvement for this set, when increasing from one to four channels, is dramatic. The average RMS-median with $C=1$ for this set of nodes is 2.54 - thus five channels achieves a $73 \%$ reduction.

Interestingly, in some cases, a lower number of channels and a lower number of nodes can actually improve performance. We do not believe that it is better, on average, to use fewer sensors or fewer channels, because we may not know ahead of time which sensor positions will be best, and we can't know ahead of time which set of channels is best. Regardless, future work may exploit adaptive methods to select channels from among those that can be measured, or adapt which set of sensors should be operating, in order to best estimate breathing rate. Such adaptive protocols could dramatically reduce the energy used in a breathing monitoring RF sensor network. Further, it would seem that better algorithms, which are more aligned with the statistics of the measured data, should be developed so that including more links' data doesn't degrade performance. 


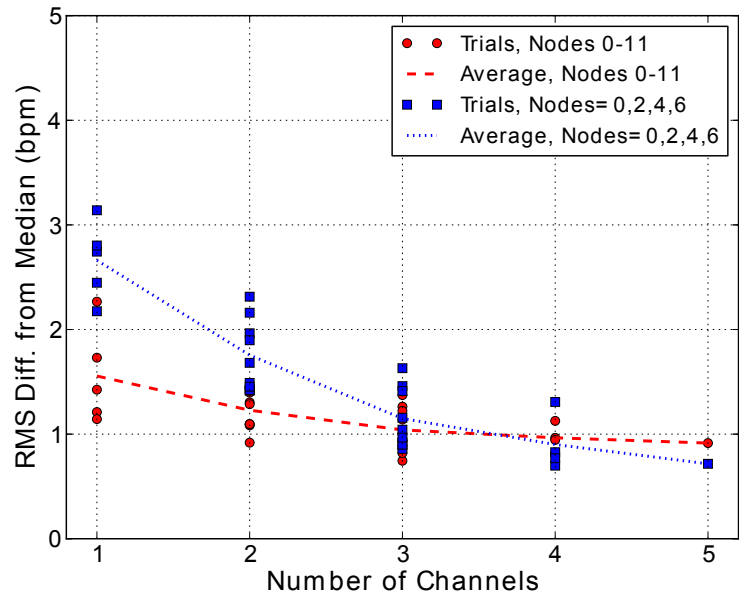

Fig. 10. RMS of the difference between estimate and short-term median vs. \# channels for Nap exp, for all twelve sensors $\{0, \ldots, 11\}$, and for node subset $\{0,2,4,6\}$.

\section{Conclusion}

This paper demonstrates key advances for the use of RSS in a wireless network for non-cooperative localization and monitoring. We show that a person's breathing alone is sufficient body movement in order to be located in a building with a static deployed wireless network. By using the estimated amplitude of breathing on each link in the network, a tomographic algorithm can produce an image map showing where breathing is occuring, and thus be used to locate a person within about 2 meters error in the experiments reported in this paper. We show that the person's breathing rate can be estimated using the same RSS measurements. When a person occasionally moves, a method described in prior work fails to accurately estimate the person's breathing rate. In this paper, a change-detection method is used to identify times at which this motion occurs, negate it, and then accurately estimate breathing rate even during short periods of motion. Methods are tested using a multi-channel sensor network deployed in two homes. The results show the possibilities for accurate and reliable breathing monitoring and localization of a breathing person for a variety of applications.

Monitoring and localizing breathing using a static wireless network has many open questions. In this paper we have assumed that breathing is periodic with a constant rate during the $N$ sample time window. However, a person's breathing may be erratic, with a changing rate over time. Further research should identify methods for estimating the duration in between two subsequent respiration peaks, from our noisy multivariate measurements, to obtain a peak-to-peak period, rather than simply a period based on the average PSD over the entire window. We may be able to do this by examining the temporal signals measured on the few links which observe high breathing amplitudes. From a signal processing perspective, this may be similar to the processing performed in pulse-position modulation (PPM) demodulators. One may then be able to identify signs of erratic breathing that may be useful for medical monitoring. Additionally, perhaps compressed sensing methods could be used to reduce the sensor and sampling requirements, and in fact, be robust to the inevitable MAC delays which will make sampling times random.

For breathing localization, we would benefit from better statistical and radio propagation models that explain a link's ability to measure breathing as a function of the person's position. What algorithms should be used to track multiple breathing people in the same deployment area? What WLAN protocols could be used to prevent an adversary from surreptitiously using someone's wireless network to eavesdrop on their breathing rate and location? How should breathing be measured with other wireless hardware, such as 802.11 devices? Each of these questions may result in interesting and useful research directions.

\section{ACKNOWLEDGEMENTS}

The authors would like to thank Brad Mager, who helped with the experiments.

\section{REFERENCES}

[1] K. Woyach, D. Puccinelli, and M. Haenggi, "Sensorless sensing in wireless networks: Implementation and measurements," in WiNMee 2006, April 2006.

[2] D. Zhang, J. Ma, Q. Chen, and L. M. Ni, "An RF-based system for tracking transceiver-free objects," in IEEE PerCom'07, 2007, pp. $135-144$.

[3] N. Patwari and P. Agrawal, "Effects of correlated shadowing: Connectivity, localization, and RF tomography," in IEEE/ACM Int'l Conf. on Information Processing in Sensor Networks (IPSN'08), April 2008, pp. 82-93.

[4] D. Zhang, J. Ma, Q. Chen, and L. M. Ni, "Dynamic clustering for tracking multiple transceiver-free objects," in IEEE PerCom'09, 2009, pp. 1-8.

[5] M. A. Kanso and M. G. Rabbat, "Compressed RF tomography for wireless sensor networks: Centralized and decentralized approaches," in 5th IEEE Intl. Conf. on Distributed Computing in Sensor Systems (DCOSS-09), Marina Del Rey, CA, June 2009.

[6] J. Wilson and N. Patwari, "See through walls: motion tracking using variance-based radio tomography networks," IEEE Trans. Mobile Computing, vol. 10, no. 5, pp. 612-621, May 2011, appeared online 23 September 2010.

[7] X. Chen, A. Edelstein, Y. Li, M. Coates, M. Rabbat, and M. Aidong, "Sequential Monte Carlo for simultaneous passive device-free tracking and sensor localization using received signal strength measurements," in ACM/IEEE Information Processing in Sensor Networks (IPSN), April 2011.

[8] C. Xu, B. Firner, Y. Zhang, R. Howard, J. Li, and X. Lin, "Improving RF-based device-free passive localization in cluttered indoor environments through probabilistic classification methods," in Proc. Information Processing in Sensor Networks (IPSN-2012), April 2012, pp. 209-220.

[9] O. Kaltiokallio, M. Bocca, and N. Patwari, "A multi-scale spatial model for RSS-based device-free localization," Arxiv.org, Tech. Rep. arXiv:1302.5914 [cs.NI], Feb. 2013.

[10] Y. Zhao and N. Patwari, "Noise reduction for variance-based device-free localization and tracking," in 8th IEEE Conference on Sensor, Mesh and Ad Hoc Communications and Networks (SECON'11), June 2011.

[11] Y. Zheng and A. Men, "Through-wall tracking with radio tomography networks using foreground detection," in Proc. Wireless Communications and Networking Conference (WCNC 2012), April 2012, pp. 3278-3283.

[12] D. Maas, J. Wilson, and N. Patwari, "Toward a rapidly deployable RTI system for tactical operations," in 8th IEEE Workshop on Practical Issues in Building Sensor Network Applications (SenseApp 2013), Oct. 2013. 
[13] M. Youssef, M. Mah, and A. Agrawala, "Challenges: device-free passive localization for wireless environments," in MobiCom '07: ACM Int'l Conf. Mobile Computing and Networking, 2007, pp. $222-229$.

[14] O. Kaltiokallio and M. Bocca, "Real-time intrusion detection and tracking in indoor environment through distributed RSSI processing," in 2011 IEEE 17th Intl. Conf. Embedded and RealTime Computing Systems and Applications (RTCSA), vol. 1, Aug. 2011, pp. $61-70$.

[15] J. Wilson and N. Patwari, "Radio tomographic imaging with wireless networks," IEEE Trans. Mobile Computing, vol. 9, no. 5, pp. 621-632, May 2010, appeared online 8 January 2010.

[16] M. Seifeldin and M. Youssef, "Nuzzer: A large-scale devicefree passive localization system for wireless environments," Arxiv.org, Tech. Rep. arXiv:0908.0893, Aug. 2009.

[17] A. Edelstein and M. Rabbat, "Background subtraction for online calibration of baseline RSS in RF sensing networks," IEEE Trans. Mobile Computing, 2012, appeared online 3 Oct. 2012.

[18] Y. Mostofi, "Compressive cooperative sensing and mapping in mobile networks," IEEE Trans. Mobile Computing, vol. 10, no. 12, pp. 1769-1784, Dec. 2011.

[19] N. Rivera, S. Venkatesh, C. Anderson, and R. Buehrer, "Multitarget estimation of heart and respiration rates using ultra wideband sensors," in 14th European Signal Processing Conference, 2006.

[20] A. Lazaro, D. Girbau, and R. Villarino, "Analysis of vital signs monitoring using an IR-UWB radar," Progress In Electromagnetics Research, vol. 100, pp. 265-284, 2010.

[21] T. Cook, N. Woodall, J. Harper, and J. Benger, "Major complications of airway management in the UK: Results of the fourth national audit project of the Royal College of Anaesthetists and the Difficult Airway Society. Part 2: Intensive care and emergency departments," British Journal of Anaesthesia, vol. 106, no. 5, pp. 632-642, 2011.

[22] A. Droitcour, O. Boric-Lubecke, and G. Kovacs, "Signal-to-noise ratio in Doppler radar system for heart and respiratory rate measurements," IEEE Transactions on Microwave Theory and Techniques, vol. 57, no. 10, pp. 2498-2507, Oct. 2009.

[23] W. Massagram, V. Lubecke, A. Host-Madsen, and O. BoricLubecke, "Assessment of heart rate variability and respiratory sinus arrhythmia via Doppler radar," IEEE Trans. Microwave Theory and Techniques, vol. 57, no. 10, pp. 2542-2549, 2009.

[24] T. Ballal, R. Shouldice, C. Heneghan, and A. Zhu, "Breathing rate estimation from a non-contact biosensor using an adaptive IIR notch filter," in 2012 IEEE Topical Conference on Biomedical Wireless Technologies, Networks, and Sensing Systems, Jan. 2012, pp. 5-8.

[25] T. Rappaport, Wireless Communications: Principles and Practice, 2nd ed. Upper Saddle River, NJ, USA: Prentice Hall PTR, 2001.

[26] W. L. Stutzman and G. A. Theile, Antenna Theory and Design. John Wiley \& Sons, 1981.

[27] J. McKenna and T. McDade, "Why babies should never sleep alone: a review of the co-sleeping controversy in relation to SIDS, bedsharing and breast feeding," Paediatr Respir Rev., vol. 6, no. 2, pp. 134-152, June 2005.

[28] N. Patwari, J. Wilson, S. Ananthanarayanan P.R., S. K. Kasera, and D. Westenskow, "Monitoring breathing via signal strength in wireless networks," arXiv.org, Tech. Rep. arXiv:1109.3898v1 [cs.NI], Sept. 2011. [Online]. Available: http://arxiv.org/abs/1109.3898

[29] J. F. Murray, The Normal Lung: The Basis for Diagnosis and Treatment of Pulmonary Disease, 2nd ed. W.B. Saunders Co., 1986.

[30] M. Basseville and I. V. Nikiforov, Detection of Abrupt Changes: Theory and Application. New Jersey: Prentice Hall, 1993.

[31] J. J. Pignatiello and G. C. Runger, "Comparisons of multivariate CUSUM charts," Journal of Quality Technology, vol. 22, no. 3, pp. 173-186, 1990.

[32] A. N. Shiryaev, Optimal stopping rules. Springer, 2007, vol. 8.

[33] S. M. Kay, Fundamentals of statistical signal processing, Volume II: Detection theory. New Jersey: Prentice Hall, 1993.

[34] H. Hashemi, "A study of temporal and spatial variations of the indoor radio propagation channel," in PIMRC-94, vol. 1, Sep 1994 , pp. $127-134$.
[35] J. Wilson and N. Patwari, "A fade level skew-Laplace signal strength model for device-free localization with wireless networks," IEEE Trans. Mobile Computing, vol. 11, no. 6, pp. 947958, June 2012, appeared online 12 May 2011.

[36] F. Wilcoxon, "Individual comparisons by ranking methods," Biometrics Bulletin, vol. 1, no. 6, pp. 80-83, 1945.

[37] D. Kifer, S. Ben-David, and J. Gehrke, "Detecting change in data streams," in Proc. 30th VLDB Conference, 2004, pp. 180191.

[38] W. J. Conover, Practical Nonparametric Statistics, 3rd ed. Wiley, 1999

[39] B. D. Ripley, Spatial Statistics. Wiley Series in Probability and Mathematical Statistics, 1981.

[40] Y. Zhao and N. Patwari, "Robust estimators for variancebased device-free localization and tracking," arXiv.org, Tech. Rep. arXiv:1110.1569 [cs.NI], Sept. 2011. [Online]. Available: http://arxiv.org/abs/1110.1569

[41] Texas Instruments. A USB-enabled system-on-chip solution for 2.4 GHz IEEE 802.15.4 and ZigBee applications. [Online]. Available: http://www.ti.com/lit/ds/symlink/cc2531.pdf

[42] O. Kaltiokallio, M. Bocca, and N. Patwari, "Follow @grandma: long-term device-free localization for residential monitoring," in 7th IEEE International Workshop on Practical Issues in Building Sensor Network Applications (SenseApp 2012), October 2012.

[43] D. P. White, J. V. Weil, and C. W. Zwillich, "Metabolic rate and breathing during sleep," J. Appl. Physiol., vol. 59, pp. 384-391, 1985

[44] Y. Katayose, M. Tasaki, H. Ogata, Y. Nakata, K. Tokuyama, and M. Satoh, "Metabolic rate and fuel utilization during sleep assessed by whole-body indirect calorimetry," Metabolism Clinical and Experimental, vol. 58, pp. 920-926, 2009.

[45] N. J. Douglas, D. P. White, C. K. Pickett, J. V. Weil, and C. W. Zwillich, "Respiration during sleep in normal man," Thorax, vol. 37, pp. 840-844, 1982.

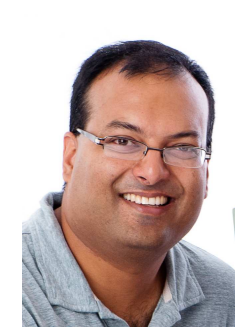

Neal Patwari received the B.S. (1997) and M.S. (1999) degrees from Virginia Tech, and the Ph.D. from the University of Michigan, Ann Arbor (2005), all in Electrical Engineering. He was a research engineer in Motorola Labs, Florida, between 1999 and 2001. Since 2006, he has been at the University of Utah, where he is an Associate Professor in the Department of Electrical and Computer Engineering, with an adjunct appointment in the School of Computing. He directs the Sensing and Processing Across Networks (SPAN) Lab, which performs research at the intersection of statistical signal processing and wireless networking. Neal is the Director of Research at Xandem, a Salt Lake City-based technology company. His research interests are in radio channel signal processing, in which radio channel measurements are used to benefit security, networking, and localization applications. He received the NSF CAREER Award in 2008, the 2009 IEEE Signal Processing Society Best Magazine Paper Award, and the 2011 University of Utah Early Career Teaching Award. He is an associate editor of the IEEE Transactions on Mobile Computing.

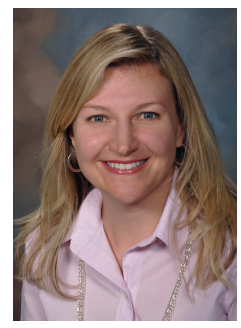

Lara Brewer received M.S. (2001) and PhD (2011) Bioengineering degrees from the University of Utah. She was a research engineer for Axon Medical between 2001 and 2006. Since 2011, she has been a Research Assistant Professor at the University of Utah. Lara's research interests are in clinical decision support and alarm systems for patient monitoring, pharmacodynamic interactions, cardiopulmonary physiology, and modeling gas exchange. 


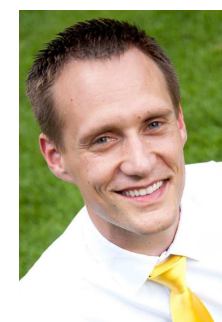

signal processing.

Quinn Tate received the B.S. and M.S. degrees from the University of Utah (2011) in Electrical and Computer Engineering where his focus of study was signal processing and electromagnetics. He has worked as a research assistant at the Utah Center of Advanced Imaging Research (UCAIR) and the Sensing and Processing Across Networks (SPAN) Lab. Quinn is currently attending the University of Utah School of Medicine where he remains active in research of biomedical applications of

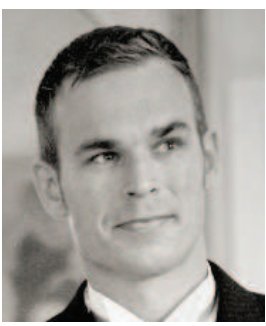

Ossi Kaltiokallio Ossi Kaltiokallio received the B.Sc and M.Sc degrees in electrical engineering from the Department of Automation and Systems Technology, Aalto University School of Electrical Engineering, Helsinki, Finland, both in 2011. He is currently a Ph.D. student with the Department of Communications and Networking, Aalto University School of Electrical Engineering. He is a member of the Wireless Sensor Systems Group at Aalto University. His current research interests include wireless communication and the indoor propagation channel, signal processing, and real-world wireless network deployments.

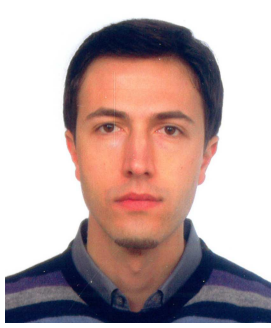

Maurizio Bocca received the B.Sc. (2003) and M.Sc. (2006) degrees in Computer Science Engineering from the Politecnico di Milano (Milan, Italy), and the Ph.D. (2011) in Electrical Engineering from Aalto University (Helsinki, Finland). In 2012, he joined as a post doc the Sensing and Processing Across Networks (SPAN) Lab at the University of Utah (Salt Lake City, Utah, USA), where he is conducting research in the area of RF sensor networks for indoor device-free localization, context awareness and elder care applications. 